Kompass

Autoimmun

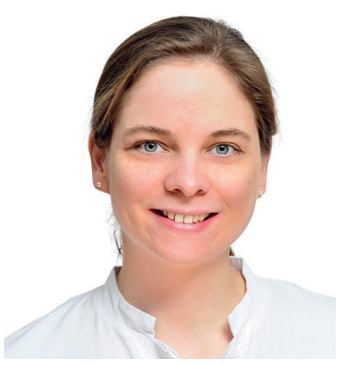

\title{
Lupusnephritis: Protokollbiopsien therapeutisch nutzen
}

\author{
Sibylle von Vietinghoff
}

Klinik für Nieren- und Hochdruckerkrankungen, Medizinische Hochschule Hannover, Hannover, Deutschland

Abstract aus Malvar A, Alberton V, Lococo B, et al.: Kidney biopsy-based management of maintenance immunosuppression is safe and may ameliorate flare rate in lupus nephritis. Kidney Int. 2020;97:156-162.

\section{Keywords}

kidney biopsy · lupus · proliferative lupus nephritis

\begin{abstract}
The optimal duration of maintenance immunosuppressive therapy for patients with lupus nephritis who have achieved clinical remission has not been established. Furthermore, clinical and histologic remissions are often discordant. We postulated that continuing therapy for patients with persistent histologic activity on kidney biopsies done during maintenance and discontinuing therapy only for patients without histologic activity would minimize subsequent lupus nephritis flares. To test this, a cohort of 75 prospectively-followed patients with proliferative lupus nephritis was managed using kidney biopsies performed during maintenance therapy. These patients had been on immunosuppression for at least 42 months, had responded, and had maintained their clinical response for at least 12 months before the kidney biopsy
\end{abstract}

was repeated. Maintenance therapy was withdrawn if the biopsy showed an activity index of zero, but was continued if the biopsy showed an activity index of one or more. A lupus nephritis flare developed in seven patients during the average 50 months from the third biopsy and the final clinic visit for a flare rate of 1.5/year; significantly less than reported flare rates. Baseline clinical parameters (serum creatinine, proteinuria) and serologic parameters (complement C3, C4 and anti-dsDNA) did not predict an activity index of zero on the third biopsy or who would have a lupus nephritis flare. No patients developed end-stage kidney disease. Four patients developed de novo chronic kidney disease. There were no serious adverse events related to biopsy. Thus, at an experienced center, biopsy-informed management of maintenance immunosuppression is safe and may improve the lupus nephritis flare rate compared to conventional clinical management.

(c) 2019 International Society of Nephrology 


\section{Transfer in die Praxis}

\section{Hintergrund}

Beim systemischen Lupus erythematodes (SLE) bestimmt die Nierenbeteiligung die Prognose entscheidend [1, 2]. Zusammen mit den Retentionswerten im Serum können Urinprotein- und Urinsedimentanalyse einen Hinweis auf eine Nierenbeteiligung bieten. Pathologisch veränderte Erythrozyten (Akanthozyten) und Erythrozytenzylinder, das sogenannte «aktive Sediment», sind typisch für inflammatorische Infiltrate in den Glomeruli. In der Niere kann das Befallsmuster, wie auch in allen anderen Organen, stark wechseln. Eine präzise Diagnose insbesondere der proliferativen Formen (WHO-Klassen III und IV) ist die Voraussetzung für eine gezielte Therapie. Das Ausmaß der Urinbefunde und die Histologie korrelieren bei der Lupusnephritis aber nur schlecht.

So ist eine Nierenbiopsie die wichtigste Richtschnur der Therapieplanung. Eindrucksvoll zeigt zum Beispiel eine dänische Kohortenstudie, dass eine frühe Biopsie die weitere Prognose beim SLE entscheidend verbessert [3]. Bei allen 91 Patienten wurde die immunsuppressive Therapie nach Befundeingang geändert. Ein langer Zeitraum (sechs Monate und mehr) zwischen dem ersten Hinweis auf renale Aktivität und der Nierenbiopsie war ein signifikanter Prädiktor für eine terminale Niereninsuffizienz. Eine Nierenbiopsie ist immer mit dem Risiko einer Blutung, die lebensbedrohlich sein oder zum Verlust einer Niere führen kann, und mit Ausfallzeiten in Beruf und Familienleben verbunden. Deswegen stellt sich die Frage, ob und wann Biopsien wiederholt werden sollten.

\section{Ergebnisse der Studie}

In der aktuellen Arbeit wurde bei Patienten mit proliferativer Lupusnephritis (62\% Klasse IV, 20\% Klasse III, 18\% Kombination von Klasse III oder IV mit Klasse V), die nach 42 Monaten Therapie eine komplette Remission oder einen klinisch stabilen Zustand erreicht hatten, eine Nierenbiopsie durchgeführt. GFR und Proteinurie sind nicht genannt. Zur Induktionstherapie waren etwa bei der Hälfte der Patienten Cyclophosphamid, bei der anderen Mycophenolat eingesetzt worden, die Erhaltungstherapie erfolgte bei fast allen (96\%) mit Mycophenolat.

Insgesamt war die Nierenbiopsie ein sicheres Verfahren. Kein Patient benötigte einen chirurgischen Eingriff oder eine Bluttransfusion. Hämatome, die analgetisch therapiert wurden, und Hämaturie waren häufig (10\% und 12\%).

Bei 72\% der Patienten war der histologische Aktivitätsindex negativ und ihre Immunsuppression wurde beendet. Bei 28\% wurde sie fortgesetzt, hier bei ca. drei Viertel unverändert mit Mycophenolat. Die anderen erhielten zusätzlich Rituximab und in einem Fall Cyclophosphamid.
Mit nichtinvasiven klinischen, laborchemischen und immunologischen Markern zum Zeitpunkt der Biopsie oder den Ergebnissen der Vorbiopsien konnte das Biopsieergebnis nicht vorhergesagt werden. Todesfälle oder terminale Niereninsuffizienz traten nicht auf. Wenn die Autoren diese Ergebnisse mit einer ähnlichen Kohorte vergleichen, war die Schubfrequenz mit 1/50 Patientenjahre deutlich niedriger als die vorher berichteten 1/6,5 Patientenjahre. Die aktuellen Ergebnisse korrespondieren mit einer 2018 publizierten kontrollierten Studie aus demselben Zentrum, bei der nach 36 Monaten die Immunsuppression bei allen klinisch stabilen Patienten abgesetzt wurde, alle aber eine Protokollbiopsie erhielten [4]. Lupusnephritisschübe in den folgenden 24 Monaten wurden auch dort bis auf einen Fall nur bei Patienten mit histologischer Aktivität beobachtet. Die aktuelle Studie geht darüber hinaus, indem sie einen Weg aufzeigt, die Ergebnisse der Protokollbiopsien therapeutisch zu nutzen.

\section{Fazit für die Praxis}

Die optimale Therapiedauer einer Lupusnephritis ist nicht definiert. In der hier untersuchten Kohorte waren Protokollnierenbiopsien eine erfolgreiche Maßnahme, um die Schubfrequenz nach Therapieänderung zu reduzieren. Die Daten stammen allerdings aus einem einzigen Zentrum in Argentinien.

Insbesondere bei komplexen Verläufen und Therapieschemata sollte eine eher großzügige Indikationsstellung für Verlaufsnierenbiopsien bei der Lupusnephritis erwogen werden.

\section{Disclosure Statement}

Hiermit erkläre ich, dass keine Interessenskonflikte in Bezug auf den vorliegenden Kommentar bestehen.

\section{Literatur}

1 Davidson A, Aranow C, Mackay M: Lupus nephritis: challenges and progress. Curr Opin Rheumatol. 2019;31:682-688.

-2 Anders HJ, Saxena R, Zhao MH, et al.:Lupus nephritis. Nat Rev Dis Primers. 2020;6:7.

3 Faurschou M, Starklint H, Halberg P, et al.: Prognostic factors in lupus nephritis: diagnostic and therapeutic delay increases the risk of terminal renal failure. J Rheumatol. 2006;33:1563-1569.

4 De Rosa M, Azzato F, Toblli JE, et al.: A prospective observational cohort study highlights kidney biopsy findings of lupus nephritis patients in remission who flare following withdrawal of maintenance therapy. Kidney Int. 2018;94:788-794.

Kontaktadresse: Prof. Dr. Sibylle von Vietinghoff, Klinik für Nierenund Hochdruckerkrankungen, Medizinische Hochschule Hannover, Carl-Neuberg-Straße 1, 30625 Hannover, Deutschland,

vonVietinghoff.Sibylle@mh-hannover.de 